\title{
Implementation of Ketogenic Diet Therapy in Refractory Epilepsies
}

\author{
Jianxiang Liao ${ }^{1}$ and Lan Xiong ${ }^{2^{*}}$ \\ ${ }^{1}$ Shenzhen Children's Hospital Epilepsy Center, Shenzhen, China \\ ${ }^{2}$ Research Centre, University Institute in Mental Health of Montreal, Montreal, Canada
}

*Corresponding author: Lan Xiong, Research Centre, University Institute in Mental Health of Montreal, Montreal, Canada, Tel: 514-251-4015; E-mail: lan.xiong@umontreal.ca

Received date: July 05, 2016; Accepted date: July 29, 2016; Published date: Aug 02, 2016

Copyright: @ 2016 Liao J, et al. This is an open-access article distributed under the terms of the Creative Commons Attribution License, which permits unrestricted use, distribution, and reproduction in any medium, provided the original author and source are credited.

\begin{abstract}
The Ketogenic Diet (KD) has been used in treatment of epilepsy in mainland China since 2004. Clinical indications of KD include: Glucose Transporter Type 1 (GLUT-1) deficiency, Pyruvate Dehydrogenase Deficiency (PDHD, myoclonus-astatic epilepsy (Doose syndrome), tuberous sclerosis complicated with or without epilepsy, Rett syndrome, Dravet syndrome, infantile spasms, and Landau-Kleffner syndrome, Lafora disease, and super-refractory status epilepticus. The contraindications of KD include: Inborn error of lipid metabolism, porphyria, and patients who are unable to cooperate with the KD. There should be standardized clinical consultation and evaluation before starting KD treatment; and special attention should be paid to selection and preparation of food, and to indication of age and geographic area etc. During the KD treatment, the transition time from ordinary diet to KD often takes 1-2 weeks; and a final 2: 1-4: 1 ketogenic diet ratio will normally produce ketosis of clinical therapeutic effect. The KD could be combined with anticonvulsant treatment. A qualified ketogenic diet therapy means: (1) Proper nutrition and growth with normal nutrition biomarkers; (2) Tasty foods: patients are willing to accept the therapeutic diet; (3) Ideal state of ketosis: urine ketone remains above $(+++)$, blood ketone at about $4.0 \mathrm{mmol} / \mathrm{L}$, blood sugar is controlled at $4.0 \mathrm{mmol} / \mathrm{L}$, ratio of blood sugar/blood ketone is about 1: 1-2: 1 ; (4) Reasonable balanced food composition, defecate daily and naturally without constipation; (5) No remarkable complication(s). It is recommended that KD could be tried at least for three months continuously. Good responders should maintain the KD therapy for 2 yrs. or so. It often needs to take 3-6 months to return back to a regular diet. KD therapy should be monitored with close follow-ups and assessments. Our extensive experience has confirmed that it is safe in clinical practice.
\end{abstract}

Keywords: Ketogenic diet; Refractory epilepsy; Complications; Qualified ketogenic diet

\section{Introduction}

Ketogenic Diet $(\mathrm{KD})$ is one kind of formula diet with high proportion of fat, low proportion of carbohydrate, appropriate protein and other nutrients. Proportion of KD means the ratio of mass weight of fatty foods to both carbohydrates and proteins, such as a ratio of 2:1, $3: 1,4: 1$, etc. Although KD has been used in the treatment of epilepsy in the United States for more than 90 yrs [1], it was only started in mainland China in 2004 [2-3].

\section{The indications and contraindications of KD therapy}

In our clinical practice, $20-30 \%$ of epilepsy patients are medically refractory, and among whom, no more than $3 \%$ will become seizurefree within one year by trying other anticonvulsant medications [4]. However, KD therapy could reach about 20\% seizure-free rate in patients with refractory epilepsy; particularly for infantile spasms, seizure-free rate could be above $38 \%$. Therefore, KD should be considered as one of the major therapeutic choices in patients with medically refractory epilepsy $[5,6]$. KD therapy indications are shown in Table 1 [6]. For some special epilepsy syndromes, such as Dravet syndrome, infantile spasms, myoclonus atonic epilepsy, and tuberous sclerosis with intractable epilepsy, KD treatment should be considered earlier if antiepileptic drug treatment initially failed and patients cannot be operated.

\begin{tabular}{|c|c|}
\hline $\begin{array}{l}\text { Diseases, } K D \text { as first line } \\
\text { therapy }\end{array}$ & $\begin{array}{l}\text { Glucose transporter type } 1 \text { (GLUT-1) } \\
\text { deficiency, pyruvate dehydrogenase deficiency } \\
\text { (PDHD) }\end{array}$ \\
\hline $\begin{array}{l}\text { Epilepsies, KD as preferred } \\
\text { therapy }\end{array}$ & $\begin{array}{l}\text { Infantile spasms, Doose syndrome; tuberous } \\
\text { sclerosis complex with epilepsy, Dravet } \\
\text { syndrome }\end{array}$ \\
\hline $\begin{array}{l}\text { Other epilepsies, KD as } \\
\text { optional therapy }\end{array}$ & $\begin{array}{l}\text { Landau-Kleffner syndrome, Lafora disease, } \\
\text { other refractory epilepsies, super-intractable } \\
\text { status epilepticus, epilepsy with continuous } \\
\text { spike and slow wave during slow wave } \\
\text { sleep(CSWS), some mitochondria diseases } \\
\text { with epilepsy, e.g., respiratory chain enzyme } \\
\text { complex I or II defects, complex I/IV defects, } \\
\text { complex IV defects }\end{array}$ \\
\hline $\begin{array}{l}\text { Other diseases, } \mathrm{KD} \text { as optional } \\
\text { therapy }\end{array}$ & $\begin{array}{l}\text { Autism, Rett syndrome, type } V \text { glycogen } \\
\text { storage disorder, subacute sclerosing } \\
\text { panencephalitis, fructose } 1,6 \text { bisphosphatase } \\
\text { deficiency }\end{array}$ \\
\hline $\begin{array}{l}\text { Diseases, KD could } \\
\text { considered as alternative }\end{array}$ & $\begin{array}{l}\text { Various inflammatory diseases, tumors, } \\
\text { obesity, diabetes mellitus, encephalitis, insult } \\
\text { of brain and spinal cord, behavioral problems } \\
\text { like impulsivity, aggression, depression, } \\
\text { schizophrenia, and neurodegenerative } \\
\text { diseases }\end{array}$ \\
\hline
\end{tabular}

Table 1: Indications of KD [6]. 
Page 2 of 5

Contraindications of $\mathrm{KD}$ therapy include porphyria, pyruvate carboxylase deficiency disease, inborn errors of fatty acid transport and mitochondrial oxidation, and some other mitochondrial diseases like pyruvate carboxylase deficiency.

\section{Nutrition evaluation and diet consultation}

Before KD treatment, a comprehensive evaluation needs to be performed, which mainly includes communication with parents evaluation of children's nutritional condition, laboratory examinations and other selective auxiliary examination etc., as shown in Table 2.

\begin{tabular}{|c|c|}
\hline Consultation & $\begin{array}{l}\text { Expectations of treatment are } \\
\text { discussed, including the seizure } \\
\text { reduction, drug therapy and } \\
\text { improvement of cognitive function; } \\
\text { understanding the possible problems } \\
\text { in KD treatment, such as the } \\
\text { implementation difficulties, the } \\
\text { economic burden, adverse reactions, } \\
\text { etc.; understanding the sugar content } \\
\text { of antiepileptic drugs and other } \\
\text { commonly used drugs; } \\
\text { recommendations of learning materials } \\
\text { to parents about KD }\end{array}$ \\
\hline Nutrition evaluation & $\begin{array}{l}\text { Height and weight baseline data, as } \\
\text { well as the patient's height and weight } \\
\text { based on the ideal BMI (body mass/ } \\
\text { height square); diet history: Record of } \\
\text { foods of the last } 3 \text { days, usual taste } \\
\text { preferences, bad eating habits, } \\
\text { anorexia and food intolerance; to } \\
\text { determine the diet formula form and } \\
\text { mode of intake: via oral or intestinal } \\
\text { tract, or both, or partially parenteral; to } \\
\text { determine how to initiate KD, e.g., by } \\
\text { medium chain triglycerides diet, or } \\
\text { classic KD, or modified Atkins diet, or } \\
\text { low-glycemic index treatment; to } \\
\text { calculate the calorie, liquid quantity } \\
\text { and the proportion of KD; to add other } \\
\text { appropriate nutritional supplements } \\
\text { based on the dietary absorption }\end{array}$ \\
\hline Laboratory tests & $\begin{array}{l}\text { Complete blood counts; electrolytes } \\
\text { and trace elements; serum } \\
\text { bicarbonate, calcium, zinc, selenium, } \\
\text { magnesium, potassium, sodium; and } \\
\text { phosphate; liver and kidney functions } \\
\text { (including blood aspartate } \\
\text { transaminase, alanine } \\
\text { aminotransferase, uric acid, total } \\
\text { protein, albumin, globulin, urea } \\
\text { nitrogen and creatinine, etc.); fasting } \\
\text { blood lipids (including high density } \\
\text { cholesterol, triglyceride, cholesterol, } \\
\text { low density cholesterol); routine urine } \\
\text { examination; urinary calcium and } \\
\text { creatinine ratio (if necessary); } \\
\text { antiepileptic drug blood concentration } \\
\text { (if necessary); serum acylcarnitine (to } \\
\text { exclude certain inherited metabolic } \\
\text { diseases); urine organic acids and } \\
\text { serum amino acid (to exclude certain } \\
\text { genetic metabolic disorders) }\end{array}$ \\
\hline Auxiliary examination (optional) & $\begin{array}{l}\text { Ultrasound of the kidney and kidney } \\
\text { disease diagnosis (especially when } \\
\text { having a family history of kidney } \\
\text { stones); electroencephalogram (EEG); } \\
\text { brain magnetic resonance imaging; } \\
\text { cerebrospinal fluid (when the etiology } \\
\text { is unclear); electrocardiogram(ECG) (if } \\
\text { any family history of heart disease) }\end{array}$ \\
\hline
\end{tabular}

Table 2: Content of evaluation and consultation before KD initiation.

\section{Different types of KD and applicable ages}

Recent practice has shown that KD therapy is suitable for patients of all ages, including adults with epilepsy $[7,8]$. KD therapy can use the already-made commercial $\mathrm{KD}$ foods, or specially prepared foods according to the principle of $\mathrm{KD}$ therapy. Babies can use the liquid product of KD formula since they cannot eat solid food; and baby KD formula also contains other essential nutrients. The liquid KD foods are used to supply nutrients to patients with tube feeding. Classic KD formula is suitable for young children, but often difficult to be to accepted by older children.

Modified Atkins Diet (MAD) and Low-Glycemic Index Treatment (LGIT) are more likely to be accepted by older children and adults. In general, for new cases it is suggested to use the already made KD foods for 3 months because of its standardized contents and formula. Starting a new diet therapy for children need to proceed carefully and adjust timely according to responses. Good responders of KD and the family who have certain experience in $\mathrm{KD}$, can use ordinary food prepared by themselves, and maintain on the KD for a long period.

In developed countries, epilepsy center or diet therapy clinic can offer KD service. But in the areas lack of such professional resources, there could be certain difficulties. In 2015, the International League against Epilepsy Dietary Therapy Task Force (ILAE, www.ilae.org) published KD treatment guideline in resource-poor regions; and suggested that the adult and pediatric neurologists should be familiar with KD treatment, and should form a special KD treatment team in resource-poor region. The classic KD therapy must have a dietitian to participate in the team [9].

Basic laboratory tests include blood sodium, potassium, bicarbonate, chloride, urea nitrogen, creatinine, and sugar. Follow-up examinations should include fasting blood lipids and urine analysis. All patients should receive a variety of vitamins and calcium screening tests. Babies or patients with comorbidities should be admitted in the hospital to initiate the KD therapy. Low-risk patients could start KD at out-patient clinics. All patients with KD treatment must be ensured that they would be able to contact the KD treatment team immediately in any emergency situation.

\section{The protocol of KD therapy is divided into five phases}

Diet initiation phase: There are two ways of diet transition: (i) Gradual increase of calories: First calculating daily calorie need, and starting from fasting, or a small amount of calories, to $1 / 3$, and to $2 / 3$ amount and then to the full amount of calculated calories; (ii) Gradual increase of the KD diet ratio, from 1:1 to 2:1, 3:1, until 4:1.

$\mathrm{KD}$ can be initiated according to the patient's individual condition, such as age, food preference, financial situation, education background and other factors. If rapid control of seizures is needed, fasting at the beginning of the therapy is appropriate, but attention must be paid with close monitoring in order to avoid low blood sugar and other adverse events. Normally the transition from ordinary diet to KD should be completed in 1-2 weeks either with hospitalization or with out-patient service, mainly to teach patients and/or parents to implement $\mathrm{KD}$. Classical KD treatment must be supervised by the experienced physician and/or dietitian who have been properly trained in KD therapy. Modified Atkins diet and LGIT diet can be administered in out-patient clinics. 
Generally, the starting KD diet ratio of 2:1-4:1, and it takes 1-2 weeks of transition from normal eating to $\mathrm{KD}$. Calories is generally $250-334 \mathrm{kj} / \mathrm{kg}$ body weight $(60-80 \mathrm{kcal} / \mathrm{kg}$ body weight); young children can be $376 \mathrm{kj} / \mathrm{kg}$ body weight $(90 \mathrm{kcal} / \mathrm{kg}$ body weight). Calories can be calculated to $70-85 \%$ of the recommended calorie needs of all ages. But specific calorie level appropriate for each individual patient needs to be titrated every 1-3 months. If the patient is overweight, it will make weight regress to the $50^{\text {th }}$ percentile best points by controlling the total calories. Diet ratio can be adjusted according to seizure control and nutrition profile.

Titration phase: 1-6 months, during this period to further adjust the ratio of $\mathrm{KD}$, aiming for the best outcome.

Consolidation phase: maintenance of KD treatment should be about 2 yrs, to consolidate therapeutic effect.

Discontinuation phase: seizure free lasted for $2 \mathrm{yrs}$, epileptiform discharges disappeared on EEG.

Returning to normal diet phase: within 3-6 months, gradual transition to a regular diet.

\section{Combined KD and antiepileptic drug therapy}

Although a small number of patients with KD therapy can achieve a seizure-free effect with the diet alone, in most cases, KD treatment needs to be used in combination with antiepileptic drugs to control the seizures. At the beginning of $\mathrm{KD}$ therapy, the original antiepileptic drug therapy remains the same for the first three months because it is easier to evaluate the response of diet therapy and also to avoid seizure deterioration. After 3-6 months, antiepileptic drugs could be reduced gradually with caution. Each time only one antiepileptic drug can be slowly decreased and the risk of seizure recurrence should be discussed with the patients or their guardians. Reducing phenobarbital and benzodiazepines need be to particularly careful and not to be too fast because of the rebound effect, i.e., stopping these drugs is more likely to cause recurrent seizures. The safest solution is to maintain the current effective antiepileptic drugs with seizure free for more than 1 $\mathrm{yr}$; then considering to reduce the dose of antiepileptic drugs and varieties gradually.

\section{Nutritional supplements during KD treatment}

Nutritional supplements should be provided for patients under KD therapy. Our general advice includes supplementing a variety of vitamins and minerals, as well as calcium supplements with vitamin D. Other selective nutritional supplements include oral potassium citrate (prevention of lithiasis), selenium, magnesium, zinc, phosphorus, vitamin $\mathrm{D}, \mathrm{L}$-carnitine, medium chain triglycerides (coconut oil or palm kernel oil), sodium salt ( $>1$ year old children should have the sodium salt calculated in the diet formula). All supplemental nutrients should not contain carbohydrates. Previous evidence suggests that in the process of $\mathrm{KD}$ treatment, daily supplements of vitamins and minerals should contain as low as possible carbohydrate. However, there is no clinical data to support the use of antacids or laxatives in the process of $\mathrm{KD}$ therapy. No specific clinical symptoms would suggest carnitine insufficiency; and the diagnosis mainly relies on laboratory test. Supplement of the L-carnitine is needed only when carnitine levels are low, usually from 50 to $100 \mathrm{mg} /(\mathrm{kg} . \mathrm{d})$ to start with, gradually increasing the amount, the largest daily dosage is not more than $2 \mathrm{~g} / \mathrm{d}$.

\section{Maintenance and follow-up of KD}

Nutritionists and physicians should closely follow up the patients treated with KD [10-11], and observation items in the follow-up are listed in Table 3. During the first year of KD treatment, there should be at least one out-patient care visit every three months, which is important for treatment success. It is recommended to shorten the time interval of out-patient care for younger children with high risk for nutritional deficiency. All patients should be evaluated by experienced physicians and dietitians through nutritional assessment and laboratory tests (Table 3). The clinical decision regarding $\mathrm{KD}$ and antiepileptic medication treatments should be discussed among responsible physicians and dietitians.

\section{Nutrition evaluations}

Weekly checkup of height, body weight to maintain the ideal height and weight growth rate and the right weight index; assessment of diet prescription (calories, protein and fluids) according to dietary intakes; vitamin and mineral supplement evaluation and guidance; assessment of the treatment compliance

\section{Medical evaluation (neurological)}

The outcome of KD (whether or not matched with the expectations of parents'); reduction of antiepileptic drugs (if possible); continuation of KD therapy; improvements of patient's behavior, attention, speech, movement, intelligence and cognitive function

\section{Laboratory evaluation}

Complete blood count; electrolyte: serum bicarbonate, total protein, calcium, magnesium, phosphate; liver and kidney functions (including albumin, globulin, aspartate transaminase, serum glutamic-pyruvic transaminase, blood urea nitrogen, creatinine, uric acid); fasting blood lipids; carnitine; routine urine examination; urine calcium and serum creatinine ratio; and antiepileptic drug blood concentration (if necessary)

\section{Items optional}

Serum beta-hydroxybutyric acid; zinc and selenium; kidney and liver ultrasound; bone density (dual-energy X-ray absorptiometry (DEXA) scan or ultrasonic scanning); EEG, and other neurological or oncology specialized examinations

Table 3: Suggestions for KD clinic follow-up.

\section{Quality assessments of KD therapy}

Compared with antiepileptic drug therapy, KD therapy is more complicated. Strict adherence to the diet is key for it to be successful and effective. Therefore, the patients and their parents need to be patient and cooperative; and the extended family (grandparents etc.) and people surrounding them (school friends, neighbours etc.) need to be understanding and supportive in correct way, i.e., not giving patients high-carb foods out of sympathy or "concern". Dietitian or diet treatment team needs to follow up closely. In terms of treatment effectiveness, we think it should be evaluated from the several following aspects according to our experience: (1) Appropriate nutrition and growth: height, weight indicators meet the criteria of the same age group; face skin color is healthy and the patients are in good mental state; nutrition biomarkers are normal; (2) Good food appetite: delicious and well-cooked food make the children and other age group patients happy with diet therapy; (3) Optimal state of ketosis: urine ketone remains above $(+++)$, blood ketone at about 2.0-4.0 $\mathrm{mmol} / \mathrm{L}$, blood sugar is controlled at about $4.0 \mathrm{mmol} / \mathrm{L}$, ratio of blood glucose/ ketone (GKI, glucose/ketone index) is about 1:1-2:1; (4) Reasonable food composition: With a variety of food or nutrition supplements, 
maintaining balanced nutrition, defecating daily and naturally without constipation; (5) Without remarkable complication(s).

\section{Efficacy of KD therapy}

Studies have shown that KD is effective to myoclonic seizures, focal motor seizures, atypical absence, and generalized tonic-clonic seizures, and other types epileptic seizures or epilepsy syndromes, the overall effect is $33-67 \%$ seizure reduction [12-15]. Freeman et al., [16] reported the effect of $\mathrm{KD}$ therapy in 150 patients in a prospective study. After one year, there were still $57 \%$ patients continuing on the KD; among them, $7 \%$ was seizure free, and $27 \%$ reduced by more than $90 \%$ of their seizures, and $50 \%$ patients had effect of over $50 \%$ seizure reduction. At the Johns Hopkins Medical Center, a multicenter, prospective study of 51 patients showed that KD therapy at one year, $40 \%$ patients with more than $50 \%$ seizure reduction [17]; $53 \%$ of patients discontinued the KD therapy. The possibility of continuing the KD therapy will be $80 \%$ if the seizure reduction reached $50 \%$. However, if seizure reduction was less than $50 \%$, only about $20 \%$ was able to adhere to the treatment. A South Korea multicenter prospective study of 199 cases also showed that $46 \%$ patients can maintain on the diet after one year; and among them, $41 \%$ patients with seizures reduced by more than $50 \%$, including seizure free in $25 \%$ cases; and $17 \%$ of children in the treatment got the "dramatic effect" (having seizure free within 2 weeks), which are mainly cases with infantile spasms, but no patient with complex partial seizures [18]. Kossoff et al reported 32 cases of infantile spasms treated by the KD therapy (2 cases did not receive any other treatment before), at one year $56 \%$ patients were still on the diet treatment, $46 \%$ patients with seizures reduced by more than $90 \%$, and $100 \%$ patients with seizures reduced by more than $50 \%$ [19]. In general, experts in the field consider that $\mathrm{KD}$ therapy is overall superior to the existing antiepileptic drugs. It is worth to emphasize that in particular, while controlling seizures with $\mathrm{KD}$, it also can supply the body with ketone bodies as energy source. Therefore, in case of low blood sugar level, the symptom of hypoglycemia won't appear because the body may have switched from glucose to ketone bodies as energy source. KD has played an important role in the improvement of behavior and cognitive functions. Research has shown that KD may improve the language development, mental retardation, attention deficit, impulsion, dyskinesia in some cases [20-23].

\section{Adverse reactions of KD therapy}

More than 90 years of clinical experience world-wide have indicated that KD therapy is safe; and adverse reactions can be avoided or can be alleviated by appropriate procedures without having to terminate the treatment $[6,24,25]$. The incidences of metabolic abnormalities are relatively low, e.g., hyperuricemia (high blood uric acid, 2-26\%), hypocalcaemia (2\%), hypomagnesemia (5\%), hypoproteinemia and acidosis (2-5\%) [6]. The incidence of gastrointestinal symptoms, including vomiting, constipation, diarrhea and abdominal pain, is about $12-50 \%$. Secondary carnitine deficiency could happen in some cases. Previous studies have also reported the incidence of hypercholesterolemia in KD patients, about 14-59\% [6]. The incidence of kidney stones in $\mathrm{KD}$ treatment in children was reported around 3-7\%; and usually do not need to stop the diet therapy, and rarely need lithotripsy or surgical treatment. Oral potassium citrate helps to prevent renal stone formation, thus make the urinary calculi incidence reduced by about 10 times [6].

\section{KD therapy discontinuation}

During KD therapy, if urgent stop is needed, it is still necessary to balance the diet to avoid sudden rise of blood sugar, particularly for the glucose infusion because sudden increase of high sugar may induce or aggravate seizures. Our experience has demonstrated that $\mathrm{KD}$ therapy should be considered to be stopped if it fails to control seizures after the first 3-6 months. If seizures are successfully controlled, the diet can generally be considered to be stopped after about 2 years, according to the clinical situation. However, for patients with glucose transporter type 1 (GLUT-1) deficiency and lack of pyruvate dehydrogenase (PDHD), and other inborn metabolic disorders, it is necessary to maintain the $\mathrm{KD}$ for a longer period. In patients with refractory epilepsy and other chronic diseases, the duration of $\mathrm{KD}$ therapy must be individualized according to the response to the KD diet [16-19]. For patients completely free of epileptic seizure, EEG examination and clinical assessment should be performed before stopping KD therapy. The overall incidence of seizure recurrence after discontinuation of KD is about $20 \%$. Patients have abnormal EEG with epileptiform discharges, abnormal magnetic resonance imaging findings of the brain; and patients whose etiology of epilepsy is tuberous sclerosis complex have greater risk of seizure recurrence. Usually the KD therapy should be gradually withdrawn during a period of 3-6 months.

\section{References}

1. Vezyroglou K, Cross JH (2016) Targeted Treatment in Childhood Epilepsy Syndromes. Curr Treat Options Neurol 18: 29.

2. Suo C, Liao J, Lu X, Fang K, Hu Y, et al. (2013) Efficacy and safety of the ketogenic diet in Chinese children. Seizure 22: 174-178.

3. Ketogenic Diet Study Group of the Subspecialty Group of Neurology, the Society of Pediatrics, Chinese Medical Association (2013) Prospective multicenter study on long-term ketogenic diet therapy for intractable childhood epilepsy. Zhonghua Er Ke Za Zhi 51: 276-282.

4. Kwan P, Brodie MJ (2000) Early identification of refractory epilepsy. N Engl J Med 342: 314-319.

5. Kossoff EH, Zupec-Kania BA, Amark PE, Ballaban-Gil KR, Christina Bergqvist AG, et al. (2009) Optimal clinical management of children receiving the ketogenic diet: recommendations of the International Ketogenic Diet Study Group. Epilepsia 50: 304-317.

6. Vehmeijer FO, van der Louw EJ, Arts WF, Catsman-Berrevoets CE, Neuteboom RF (2015) Can we predict efficacy of the ketogenic diet in children with refractory epilepsy? Eur J Paediatr Neurol 19: 701-705.

7. Cervenka MC, Henry BJ, Felton EA, Patton K, Kossoff EH (2016) Establishing an Adult Epilepsy Diet Center: Experience, efficacy and challenges. Epilepsy Behav 58: 61-68.

8. Schoeler NE, Cross JH (2016) Ketogenic dietary therapies in adults with epilepsy: a practical guide. Pract Neurol 16: 208-214.

9. Kossoff EH, Al-Macki N, Cervenka MC, Kim HD, Liao J, et al. (2015) What are the minimum requirements for ketogenic diet services in resource-limited regions? Recommendations from the International League against Epilepsy Task Force for Dietary Therapy. Epilepsia 56: 1337-1342.

10. Kossoff EH, Freeman JM, Turner Z, Rubenstein JE (2011) Ketogenic Diets: Treatments for Epilepsy and Other Disorders (5th edn.) New York: Demos.

11. Liao JX, Fang KW, Deng YH (2011) Ketogenic diet therapy for childhood epilepsy. People's Health Care Publishing House pp: 20-33.

12. Vining EPG (1999) Clinical efficacy of the ketogenic diet. Epilepsy Res 37: $181-190$

13. Lambrechts DA, de Kinderen RJ, Vles JS, de Louw AJ, Aldenkamp AP, et al. (2016) A randomized controlled trial of the ketogenic diet in refractory childhood epilepsy. Acta Neurol Scand. 
14. Hallböök T, Sjölander A, Åmark P, Miranda M, Bjurulf B, et al. (2015) Effectiveness of the ketogenic diet used to treat resistant childhood epilepsy in Scandinavia. Eur J Paediatr Neurol 19: 29-36.

15. Neal EG, Chaffe H, Schwartz RH, Lawson MS, Edwards N, et al. (2008) The ketogenic diet for the treatment of childhood epilepsy: a randomised controlled trial. Lancet Neurol 7: 500-506.

16. Freeman J, Vining E, Pillas D, Pyzik PL, Casey JC, et al. (1998) The efficacy of the ketogenic diet-1998: a prospective evaluation of intervention in 150 children. Pediatrics 102: 1358-1363.

17. Vining E, Freeman J, Ballaban-Gil K, Camfield CS, Camfield PR, et al (1998) A multicenter study of the efficacy of the ketogenic diet. Ach Neurol 55: 1433-1437.

18. Kang HC, Kim YJ, Kim DW, Kim HD (2005) Efficacy and safety of the ketogenic diet for intractable childhood epilepsy: Korean multicentric experience. Epilepsia 46: 272-279.

19. Kossoff EH, Pyzik PL, McGrogan JR, Vining EP, Freeman JM (2002) Efficacy of the ketogenic diet for infantile spasms. Pediatrics 109: 780-783.
20. Hong AM, Turner Z, Hamdy RF, Kossoff EH (2010) Infantile spasms treated with the ketogenic diet: prospective single-center experience in 104 consecutive infants. Epilepsia 51: 1403-1407.

21. Wu YJ, Zhang LM, Chai YM, Wang J, Yu LF, et al. (2016) Six-month efficacy of the Ketogenic diet is predicted after 3 months and is unrelated to clinical variables. Epilepsy Behav 55: 165-169.

22. Zhu D, Wang M, Wang J, Yuan J, Niu G, et al. (2016) Ketogenic diet effects on neurobehavioral development of children with intractable epilepsy: A prospective study. Epilepsy Behav 55: 87-91.

23. Cross H (2015) Epilepsy: behavioural, psychological, and ketogenic diet treatments. BMJ Clin Evid pii: 1214.

24. Lu ZY, Yu LF, Gong XY, Wang XH, Hua HM, et al. (2013) Compliance and safety of ketogenic diet therapy for childhood intractable epilepsy. Chinese J Practical Pediatrics 28: 378-382 (in Chinese).

25. Wu CF, Liao JX, Zhen G, Lu XG, Cao Dezhi (2013) Case report, ketogenic diet therapy for febrile related epilepsy syndrome. Chinese J Pract Pediatrics 28: 330-333. 